Proceedings of the 48th International School and Conference on the Physics of Semiconductors "Jaszowiec 2019"

\title{
An Influence of X-Ray Irradiation on Mid-Bandgap Luminescence of Boron Nitride Epitaxial Layers
}

\author{
M. SzoŁa*, M. Tokarczyk, G. Kowalski, J. Binder, K. PakuŁa, A. Dąbrowska, \\ A. WYSMOŁEK AND J. ŁUSAKOWSKI \\ Institute of Experimental Physics, Faculty of Physics, University of Warsaw, \\ L. Pasteura 5, PL-02093 Warsaw, Poland
}

\begin{abstract}
Samples of boron nitride $(\mathrm{BN})$ in the form of an exfoliated monolayer, epitaxial layers grown on sapphire, and a powder with micrometer grains were studied in a luminescence experiment connected with irradiation of samples with X-rays. The luminescence excited with photons with the wavelength of $488 \mathrm{~nm}$ was shown to span over a broad band from a filter cut-off at $550 \mathrm{~nm}$ to about $850 \mathrm{~nm}$. The intensity of luminescence showed a monoexponential decrease both before and after irradiation with X-rays, but this dynamics slowed down after irradiation to an extent dependent on the X-ray dose. This indicates possible application of BN in dosimetry of X-rays. An influence of sapphire substrates on photoluminescence dynamics was also studied. We show that this influence is very strong in the case of layers with thickness of a few $\mathrm{nm}$ and can be disregarded when the thickness is equal to a few tens of $\mathrm{nm}$.
\end{abstract}

DOI: 10.12693/APhysPolA.136.620

PACS/topics: Boron nitride, X-Ray, Luminescence, Dosimetry

\section{Introduction}

Boron nitride has been known since its first synthesis in 1842 , but nowadays has become one of the most intensively studied semiconductor for modern optoelectronics. One of the reasons is that its hexagonal form (h-BN) suits very well as a substrate for graphene and other two-dimensional materials $[1,2]$. This useful feature is even further strengthened by a large band gap of BN (equal to about $6.4 \mathrm{eV}$ ) which allows using it as a transparent substrate for blue optoelectronics. Boron nitride crystallizes in different forms, of which hexagonal $[3,4]$ and cubic $[5,6]$ attract the most vivid interest. In this paper we concentrate on hexagonal BN only.

There are a few reasons which directed us towards the studies presented in this paper. First, the Faculty of Physics, University of Warsaw, is one of a few experimental centres at which $\mathrm{BN}$ is grown with an metal organic chemical vapour deposition (MOCVD) and it is interesting to test its properties by different techniques. Second, some epitaxial layers exhibit a broadband photoluminescence (PL) with a spectrum covering almost the full range of visible light. This PL is tentatively attributed to defects and it is interesting to verify whether X-ray photons influence the PL; and if they do, whether the changes are temporary or permanent. This could shed some light on the nature of defects involved in the PL. Third, it was found that irradiation of BN layers with electrons leads to changes in the Raman or PL spectra and it was our aim to verify whether similar changes can be generated with $\mathrm{X}$-ray photons. Next, we wanted to check whether boron nitride could be used as a dosimetric material working on

\footnotetext{
* corresponding author
}

the principle of optically stimulated luminescence (a dosimetric method which relates changes of luminescence intensity to absorbed dose of radiation [7]). Once boron nitride-based devices become reality in future electronics, dosimetric properties of this material could be addressed to estimate radiation doses absorbed by people in massive nuclear or radiation accidents.

The basic outcome of our study is the following: the most consistent results have been obtained on powdered samples which can be proposed as a dosimetric material; the Raman spectra do not reveal changes related to irradiation with X-rays, and irradiation with X-ray changes a long-term dynamics of the lumienscence to an extent dependent on the time of exposition to X-rays.

\section{Experimental techniques}

Beside sapphire substrates, three types of BN samples were studied: an exfoliated monolayer, epitaxial layers grown with an MOCVD, and a commercially available microcrystalline powder. All samples showed a hexagonal BN structure as revealed with X-ray diffractometry. Observation of PL was carried out in an experimental system presented schematically in Fig. 1. In the case of epitaxial layers and sapphire substrates, the samples were attached to a gold-metallized support with copper wires (acting as a kind of little springs) and placed in a focal point of a laser beam. In the case of BN powder, we used aluminium plates with a drilled hollow in which the powder was placed. The exfoliated monolayer was attached to a copper plate and suspended over a hole (the diameter of $1 \mathrm{~mm}$ ) drilled in the plate. We used one of the lines of of $\mathrm{Ar}^{+}$laser with the wavelength equal to $488 \mathrm{~nm}$. The laser excitation beam passed through an interference filter which eliminated plasma lines form 

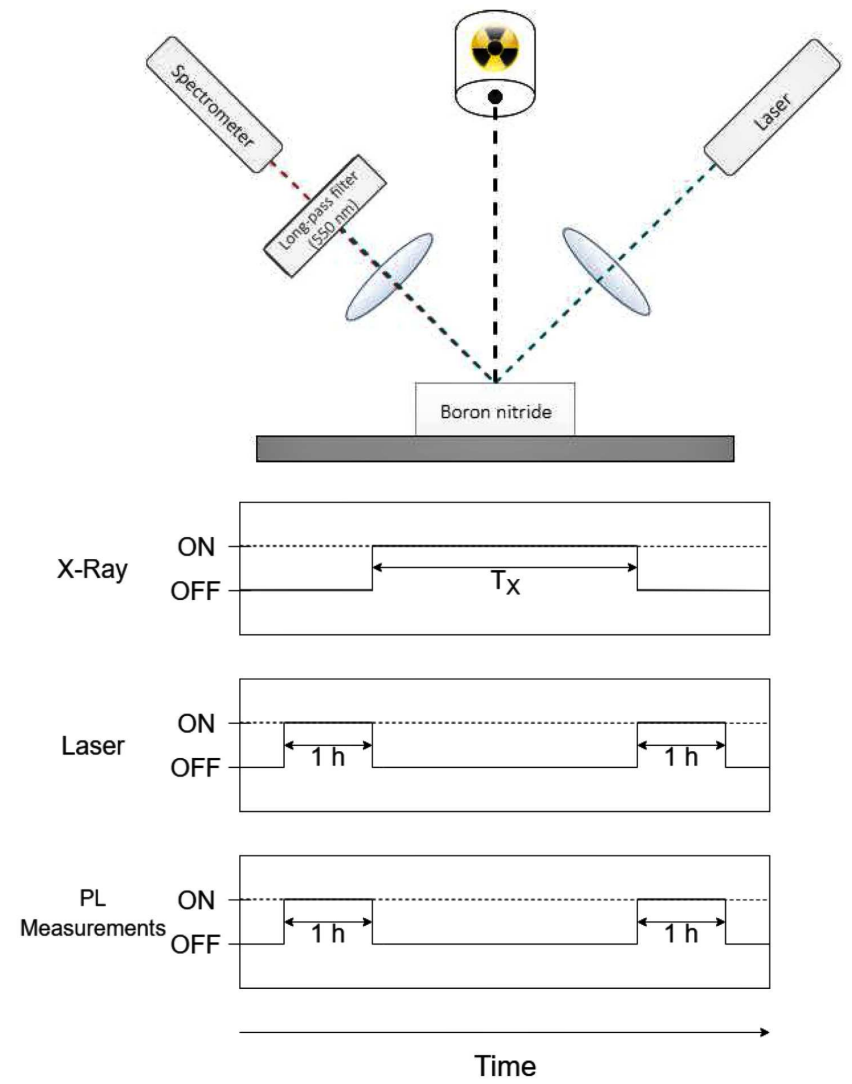

Fig. 1. A scheme of the experimental setup (top); a time sequence of luminescence-X-rays-luminescence measurements (bottom).

the exciting light. The luminescence was collected by a lens and focalized on an entrance aperture of an optical fibre. A long-pass filter (with the edge at $550 \mathrm{~nm}$ ) was used to cut-off the laser light from the spectrometer. A special care was taken to eliminate any material (plastic, glues) which could be a source of unwanted luminescence. Intensity of the laser was monitored during the experiments (by reflected a small part of the beam towards a power meter), although it was not registered. We observed that variations of the laser intensity were on the level of a few percent which cannot explain long-term changes observed in photoluminescence intensity. The luminescence was registered with an OceanOptics HR 4000 spectrometer.

The experimental system described above was constructed around an X-ray diffractometer based on a $\mathrm{Cu} K_{\alpha}$ tube which generated photons of the energy equal to $8 \mathrm{keV}$. The X-ray beam passed through a collimator and showed a diameter of about $1.5 \mathrm{~mm}$ at the sample. Once placed in the experimental system, the sample was left untouched until the end of measurements. This experimental arrangement allowed to keep the sample before and after irradiation at the same position and thus to eliminate possible changes of PL signal resulting from samples' non-uniformity.
The experimental procedure consisted of three steps. The first one was collecting the PL signal during one hour after putting the sample in the experimental system. The second one was exposing the sample to X-rays during a time $T_{X}$, with the laser off. The final one was collecting the PL signal again during one hour after X-ray tube was turned off. A diagram of the time sequence of these events is shown in Fig. 1. Luminescence spectra were collected in periods of $1 \mathrm{~min}$ or $20 \mathrm{~s}$, depending on the intensity of the signal.

This procedure was applied because after first trials we realized that the luminescence intensity decreased in time even without any X-ray irradiation. Also, in spite of the fact that we paid a high attention to keep the system as mechanically stable as possible, we were not quite sure that no accidental vibrations affect the results. Such vibrations could have been caused by circulating water (a cooling system of the tube) or by opening and closing the shutter of the tube (which was done with an electrically controlled mechanism). Thus, we decided that it is the dynamics of the luminescence decrease in time rather than its intensity which can give a reliable information about the influence of X-rays on the photoluminescence.

A general difficulty in drawing a conclusion about the influence of X-rays on the photoluminescence comes from the fact that excitation with a laser light changes the intensity of PL. That is why we had to exclude a simple experimental procedure in which one repeatedly irradiates the same sample with X-rays and excites the luminescence. For, in such a case, one could never be sure if observed effects (e.g., a decrease of the PL intensity) results from X-rays or the laser light. In such a situation, to observe an influence of longer and longer times of irradiation with $\mathrm{X}$-rays, we had to start each time with a new sample. It appeared that it was only possible in the case of BN powder because we could prepare many samples, nominally identical. That is why the most informative data refer to powder samples.

\section{Results and discussion}

We adopted the procedure described above to three epitaxial layers which differed only by the thickness $d$ of BN: $d=5 \mathrm{~nm}, 20 \mathrm{~nm}$ and $80 \mathrm{~nm}$. After preliminary measurements which gave somehow non-consistent results on such samples, we decided to test the sapphire substrates. We found that the luminescence of the substrate behaves in a way similar to BN epitaxial layers, i.e., it decreases in time of excitation with the laser beam. A careful look at $\mathrm{PL}$ results on substrates and epitaxial layers showed that for thin layers, PL is strongly influenced by the luminescence of the substrate and only for the thickest layer one could say that the observed PL comes from the layer itself. For these reasons we consider results obtained on epitaxial layers as non-conclusive. Nevertheless, we verified that the Raman spectra on epitaxial layers before and after $3 \mathrm{~h}$ of irradiation with X-rays were the same. 

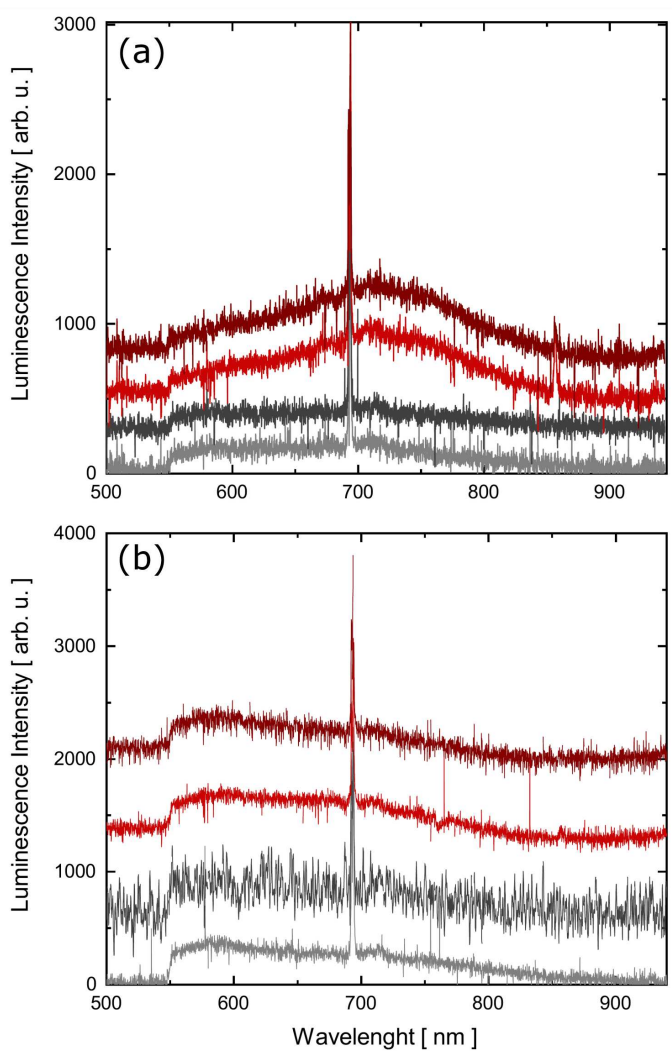

Fig. 2. (a) PL spectra on a sapphire substrate. Bottom to top: the first PL spectrum before X-rays $(T=0)$, the last PL spectrum before X-rays $(T=1 \mathrm{~h})$, the first $\left(T=1 \mathrm{~h}+T_{X}\right)$ and the last $\left(T=1 \mathrm{~h}+T_{X}+1 \mathrm{~h}\right)$ spectrum after X-rays $\left(T_{X}=3 \mathrm{~h}\right.$ in this case). The spike corresponds to a luminescence from residual $\mathrm{Cr}$ impurity. (b) PL spectra on an $80 \mathrm{~nm}$-thick epitaxial layer of $\mathrm{BN}$ on a saphire substrate. Ordering of spectra is the same as in the left part. In both figures the spectra are vertically shifted for better visibility.

As an example, we present in Fig. 2 results on a substrate which was kept in a MOCVD reactor during a thermal heating phase preceding the growth of $\mathrm{BN}$, but was removed from the reactor before $\mathrm{BN}$ growth started.

One can see that irradiation with X-rays change essentially the spectrum. Similar changes were observed on epitaxial layers on the substrate. Due to a wide band-gap of BN, epitaxial layers are transparent both to the exciting photons and to the luminescence. That is why in the case of thin epitaxial layers we observed mainly X-raysinduced changes coming from the substrate, not from a BN layer. However, a layer of $80 \mathrm{~nm}$, presented on the right in Fig. 2 seems to cover changes induced by the substrate (this was not the case for thinner layers for which changes characteristic to the substrate were observed). For this reason, future studies on epitaxial layers should be carried out on layers even thicker than $80 \mathrm{~nm}$ studied in this work.

A typical dynamics of the luminescence measured on a BN powder is shown in Fig. 3. Each point on this curve is an integral of a PL spectrum in the range 550-850 nm. Thus, the curve shows an overall dynamics of the photoluminescence. Similar curves were obtained in other cases. We found that this dynamics could be well described with a single exponent with a characteristic time $\tau$. Since the main influence of X-rays on photoluminescence was found in the value of $\tau$, we had to verify that $\tau$ did not depend on the time of measurements of the photoluminescence. The results are shown in the inset to Fig. 3, where one can check that the integral of luminescence follows a single exponential decrease over the period of at least $6 \mathrm{~h}$, a time three times longer than that used in measurements scheme described in Fig. 1.

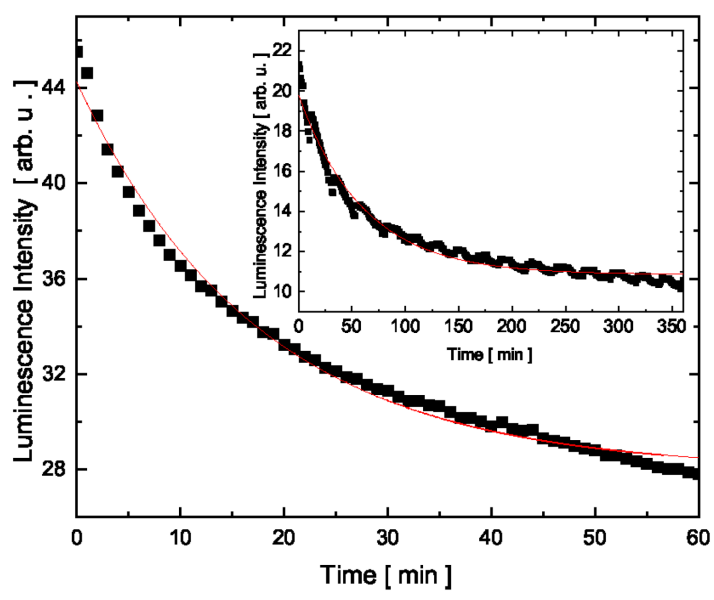

Fig. 3. A decrease of the integral of PL spectrum of a powdered sample after irradiation with X-rays during $1 \mathrm{~h}$. The inset shows a $6 \mathrm{~h}$-long dynamics of PL on a powder which was never X-rays irradiated. Thin lines show a fit with a monoexponential function.

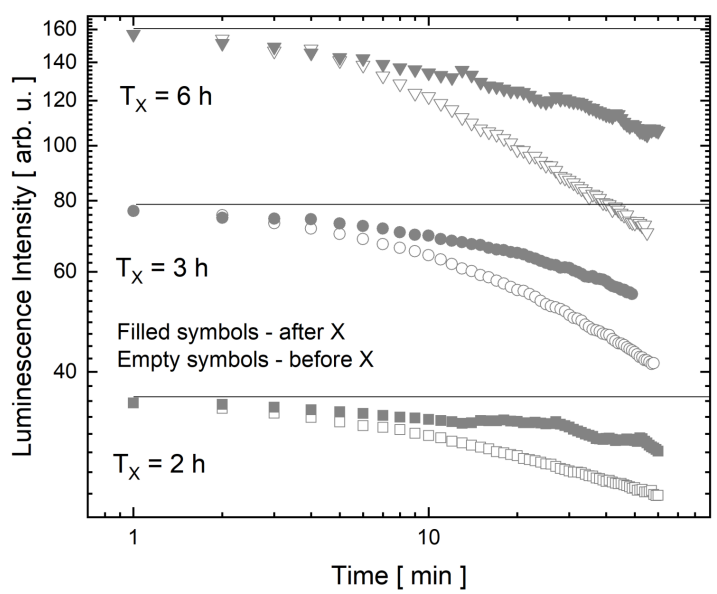

Fig. 4. A dynamics of PL in powdered samples before and after irradiation with X-rays. The time of irradiation $\left(T_{X}\right)$ is given in the figure. The values are normalized to the PL value measured during the first minute. By additional calibration measurements we estimate that during one hour of irradiation with X-rays a dose of $12 \mathrm{mGy}$ is absorbed in the powder). 


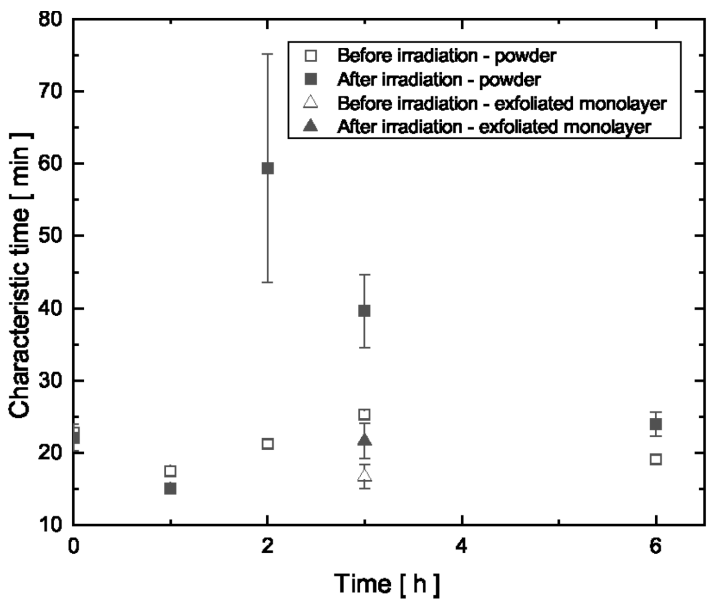

Fig. 5. Characteristic times of monoexponential decays of luminescence obtained from fitting as a function of the time of X-rays-irradiation.

A PL dynamics for different powder samples irradiated by X-rays during the time $T_{X}$ is presented in Fig. 4, while the time constants $\tau$ determined from fitting are collected in Fig. 5. One can notice that the characteristic times for the dynamics before irradiation (open squares in Fig. 5) are somehow scattered but can be approximately described with $\tau=(22 \pm 2) \mathrm{min}$. The characteristic time after irradiation does not change during the first hour but then it grows rapidly and seems to decrease to the starting value after about $6 \mathrm{~h}$ of irradiation.

Since the energy of photons used to excite the luminescence in the present experiments $(2.54 \mathrm{eV})$ is much smaller than that of the band gap of BN (equal to about $6.4 \mathrm{eV}$ ), the luminescence observed is solely related to traps (of unknown nature). The results presented in Fig. 5 suggest that X-rays first activate traps which are initially not active in PL (a rapid increase of $\tau$ ) and then deactivate them (a decrease of $\tau$ with time of irradiation). Let us also note that dynamics of the exfoliated monolayer did not change after $3 \mathrm{~h}$ of irradiation (triangles in Fig. 5) which could suggest that the monolayer is free from the traps influencing the photoluminescence in the powder.

\section{Conclusion}

We have shown that the photoluminescence from powder of $\mathrm{BN}$ is sensitive to X-ray irradiation and the best way to observe this influence is to trace dynamics of a decrease of PL with time before and after irradiation. We can propose that this material could potentially show dosimetric applications for low doses of X-rays.

\section{References}

[1] H. Nematian, M. Moradinasab, M. Pourfath, M. Fathipour, H. Kosina, J. Appl. Phys. 111, 093512 (2012).

[2] S. Lu, A.J.H. McGaughey, J. Appl. Phys. 121, 115103 (2017).

[3] K. Watanabe, T. Taniguchi, H. Kanda, Nature Mater. 3, 404 (2004).

[4] K. Zhang, Y. Feng, F. Wang, Z. Yang, J. Wang, J. Mater. Chem. C 5, 11992 (2017).

[5] L. Vel, G. Demazeau, J. Etourneau, Mater. Sci. Eng. B 10, 149 (1991).

[6] P.B. Mirkarimi, K.F. McCarty, D.L. Medlin, Mater. Sci. Eng. R Rep. 21, 47 (1997).

[7] L. Boetter-Jensen, S.W.S. McKeever, A.G. Wintle, Optically Stimulated Luminescence Dosimetry, Elsevier, 2003. 